\title{
Isolation and characterization of liver epithelial progenitor cells from normal adult rhesus monkeys (Macaca mulatta)
}

Cell Research (2009) 19:268-270. doi: 10.1038/cr.2009.9; published online 20 Jaunary 2009

\section{Dear Editor,}

Based on their ability to proliferate and the capacity to differentiate into specific cell types, hepatic progenitor/stem cells (HPCs) from adult human liver may have potential therapeutic effects on end-stage liver failure. In addition, adult HPCs have a reduced risk of teratoma formation and are not subject to the same ethical issues as fetal HPCs or embryonic stem cells [1]. The HPCs from rhesus monkeys are relevant because they may serve as a valuable preclinical model for assessment of cell therapy in humans. To date, there are no reports of HPCs or liver epithelial progenitor cells (LEPCs) isolated from normal adult rhesus monkey although a few studies in other species were reported $[2,3]$. We report here for the first time the successful isolation of rhesus monkey LEPCs (mLEPCs) from normal adult livers $(n=12)$.

Liver cell aggregates (Supplementary information, Figure S1A) used were formed from freshly digested hepatic cells, using a floating culture system [4]. Single aggregates were manually selected under a microscope and plated onto 96-well plates with one aggregate in each well. About $60 \%$ of attached aggregates consisted of elongated spindle cells with dark nuclei (Figure 1A-i), whereas about $40 \%$ of attached aggregates consisted of polygonal cells with epithelial-like morphology (putative HPCs as described [4], Figure 1A-ii). The spindle cells, which disappeared within 5 days, were identified as endothelial cells by positive staining for vimentin and brachyury (Supplementary information, Figure S1B and S1C). In contrast, polygonal epithelial cells exhibited robust proliferation ability in the medium containing $10 \%$ FBS, 50ng/ml EGF and $10 \mathrm{ng} / \mathrm{ml}$ HGF together with rat tail collagen gel.

The polygonal epithelial cells from single aggregates

Correspondence: Weizhi Ji ${ }^{\mathrm{a}}$, Qi Zhou ${ }^{\mathrm{b}}$

${ }^{a}$ Tel/Fax: +86-871-5139413; E-mail: wji@mail.kiz.ac.cn

${ }^{\mathrm{b}}$ E-mail: qzhou@ioz.ac.cn proliferated rapidly and formed moderate colonies within 4-5 days (Supplementary information, Figure S2A) and large colonies within 8-10 days (Supplementary information, Figure S2B) consisting of thousands of cells with similar morphology to previously identified HPCs $[5,6]$. Almost all of the polygonal epithelial cells in different colonies were positive for E-cad (Figure 1B-i), a marker of HPCs [7, 8]. FACS sorting was then employed to yield E-cad+CD34-CD45-CD90- cells (Supplementary information, Figure S3A-S3C), and the E-cad+ cells were enriched from $96.3 \%$ (Supplementary information, Figure S3C) to $99.8 \%$ after sorting (Figure 1C). The sorted cells rapidly formed colonies (Supplementary information, Figure S2C), stained positively for hepatic epithelial markers CK7 (Figure 1B-ii) as well as CK8/ CK18 (Figure 1B-iii), and exhibited a hepatobiliary phenotype of HPCs [9]: expression of hepatocytic (albumin, APOH) and cholangiocytic (IB4, CX43) genes as well as HNF $1 \beta$ and HNF3 $\beta$ (Figure 1B-iv). More importantly, the cells were cultured for at least 20 passages without change of expression profiles and cell morphology (data not shown). All of the above results indicated that the FACS-sorted cells might be HPCs.

Differentiation capacity was examined to assess if the E-cad+CD34-CD45-CD90- cells were progenitor cells. In hepatocytic induction medium for 10-14 days, the cells showed mature hepatocytes morphology (Figure 1D-i), expressed hepatocytic specific genes (HNF1 $\alpha$, AFP, G6P, APOB, AAT, CYP1B1 and ADH1C, Figure $1 \mathrm{D}$-vi) and protein (ALB, Figure 1D-ii), and acquired hepatocytic functions such as intracytoplasmic glycogen storage (Figure 1D-iii), ICG uptake (Figure 1D-iv) and lipid droplet generation (Figure 1D-v). When grown in 3-D collagen gels or on Matrigel for 5-7 days [10], the E-cad+CD34-CD45-CD90- cells rearranged and formed doughnut-like structures with a central lumen in 3 dimensions (Figure 1D-vii and -ix). Some cells in duct-like structures were positive for CK19 (Figure 1Dviii and $-\mathrm{x}$ ), the specific marker for adult cholangiocytes. Cholangiocytic differentiation was further confirmed by 
A

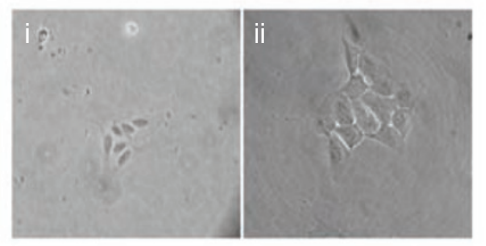

B

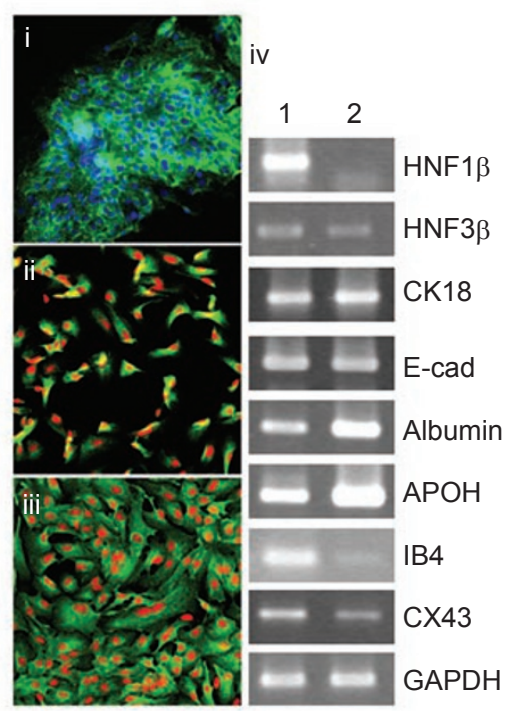

C

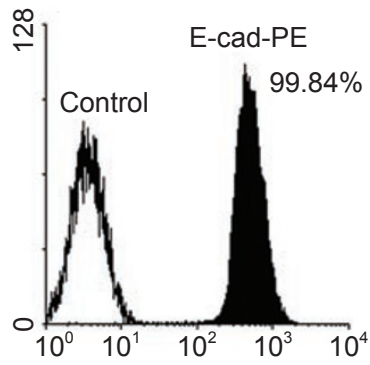

$\mathrm{D}$
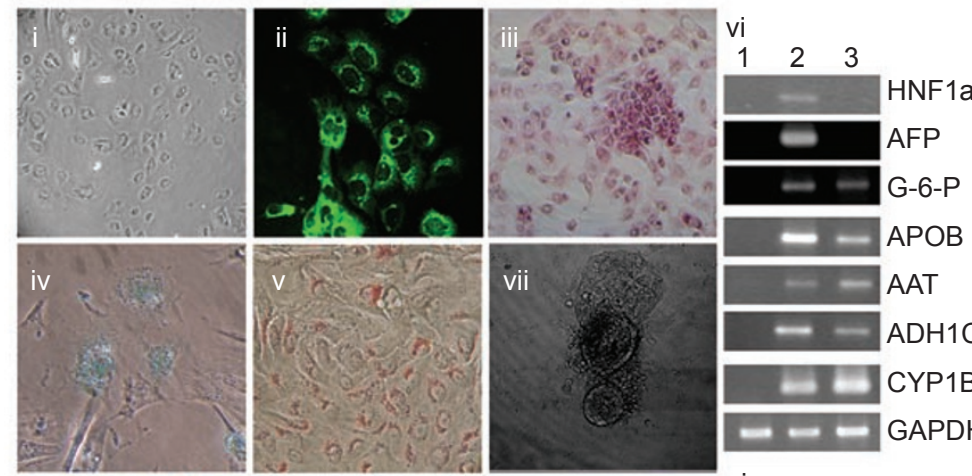

$=\mathrm{ADH} 1 \mathrm{C}$

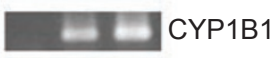

$=$ GAPDH
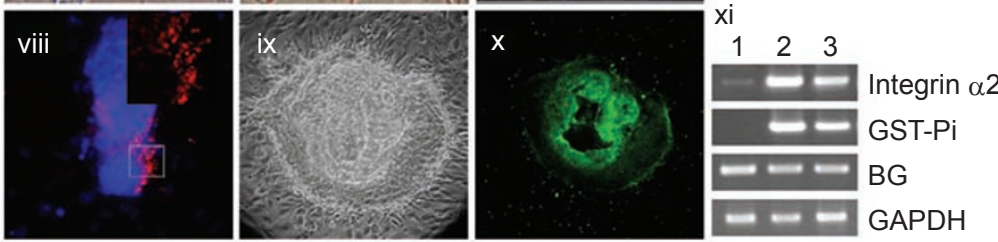

$\mathrm{E}$
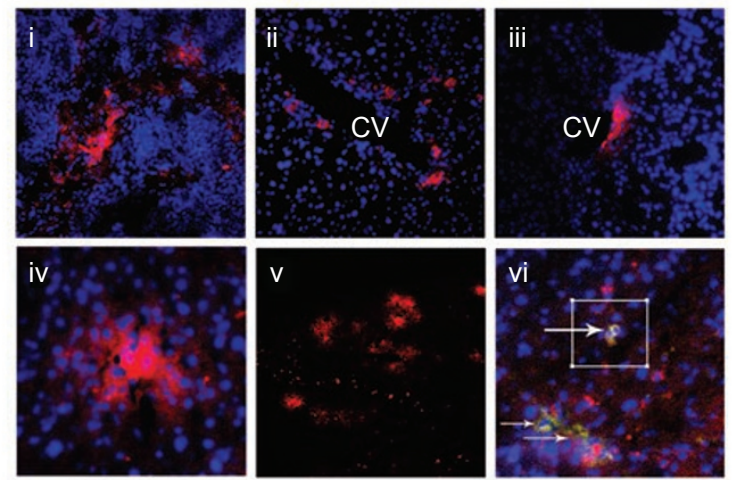

Figure 1 Isolation and characterization of liver epithelial progenitor cells from normal adult rhesus monkeys (Macaca mulatta). (A) Morphology of liver endothelial cells (i) and polygonal epithelial cells (ii) from single aggregates in different wells. (B) Immunostaining and RT-PCR analysis of polygonal epithelial cells and E-cad+CD34-CD45-CD90- sorted cells. Almost $100 \%$ of the cells in the moderate colony were positive for E-cad (green, i). FACS sorted cells from polygonal epithelial colonies were positive for CK7 (green, ii) and CK8/18 (green, iii). (iv): RT-PCR analysis of mRNA expression showed that Ecad+CD34-CD45-CD90- cells (lane 1) exhibited hepatobiliary phenotype; lane 2 was a tissue sample from adult rhesus liver. (C) FACS analysis showed that $99.8 \%$ of E-cad+CD34-CD45-CD90- sorted cells were positive for E-cad. (D) Induction of mature hepatocytes (i-vi) and cholangiocytes (vii-xi) from E-cad+CD34-CD45-CD90- sorted cells. Differentiated cells showing adult hepatocyte-like morphology (i), expression of ALB (green, ii), glycogen deposits (red, iii), ICG uptake (green, iv), and fat deposits (red, v). (vi): RT-PCR analysis of E-cad+CD34-CD45-CD90- sorted cells (lane 1), differentiated cells (lane 2), and adult rhesus liver (lane 3) showed that differentiated cells expressed hapatocytic related genes. (vii-xi) Induction of bile duct-like structures on matrigel or collagen gel coated plastic dishes. Duct-like structures (vii, ix) formed from E-cad+CD34CD45-CD90- sorted cells. Differentiated cells were positive for CK19 (red viii and green x). (xi): RT-PCR analysis of Ecad+CD34-CD45-CD90- sorted cells (lane 1), differentiated cells (lane 2), and adult rhesus liver (lane 3) showed that differentiated cells expressed cholangiocytic related genes. (E) In vivo transplantation of labeled mLEPCs into immunosuppressed mice. PKH-26 labeled cells (red) were observed in the spleen of a recipient mouse on day 1 (i). A few labeled cells migrated into the periphery of the $\mathrm{CV}$ on day 1 (ii), proliferated in the $\mathrm{CV}$ on day 7 (iii) and integrated into liver parenchyma on day 15 (iv). (v): Overall distribution of PKH-26 labeled cells in recipient liver. (vi): Staining of PKH-26 positive cells (red) with simianspecific albumin (yellow). High magnification images of cells from (vi) were showed in Supplementary information, Figure S4. Nuclear DNA was counterstained with PI (red) or Hoechst (blue). 
the expression of cholangiocyte-specific genes integrin $\alpha 2$, GST-Pi and biliary glycoprotein (BG) (Figure 1D-xi). None of above differentiated properties was observed in untreated E-cad+CD34-CD45-CD90- populations. Thus, the E-cad+CD34-CD45-CD90- populations were shown to be bipotent and were designated as monkey liver epithelial progenitor cells (mLEPCs).

PKH-26 labeled mLEPCs were injected intrasplenically into immunosuppressed mice to investigate their repopulation ability in vivo. On day 1 , many labeled cells were found in the spleen (Figure 1E-i), whereas only a few labeled cells were observed in the periphery of the central vein $(\mathrm{CV})$ in the liver (Figure 1E-ii). On day 7 , the number of the cells in the periphery of the central vein increased (Figure 1E-iii). On day 15, labeled cells integrated into the liver parenchymal region and proliferated to form cell clusters (Figure 1E-iv); the degree of repopulation was estimated to be about $2-10 \%$ (Figure $1 \mathrm{E}-\mathrm{v})$. Immunohistochemical analysis showed that less than $1 \%$ of the donor cells were positive for simian-specific albumin (Figure 1E-vi). These results showed that mLEPCs survived, proliferated and gave rise to hepatocytes in vivo.

In conclusion, isolation of mLEPCs functioning as HPCs in normal adult rhesus monkeys were reproducibly achieved from a piece of liver tissue. The purified mLEPCs displayed typical characteristics of HPCs, and differentiated into cholangiocytes and functional hepatocytes in vitro. Furthermore, mLEPCs repopulated and differentiated into hepatocytes in a liver injured mouse model. The isolation of mLEPCs has improved the knowledge of HPCs in primates, which may facilitate clinical application of HPCs in the future. Experimental materials and methods are depicted in the Supplementary information, Data S1.

\section{Acknowledgment}

We wish to acknowledge Mr Li Jian (Kunming Institute of Zoology, CAS, China) for assistance in confocal microscopic analysis. This work was supported by research grants from National 863 High Technology Research and Development Program of China 2005AA219010 and 2006AA02A116, National Basic Research Program of China 2006CB701505 and 2007CB947701, The Chinese Academy of Sciences KSCX1-YWR-47 and KSCX105-02, and Chinese National Science Foundation 30700159 and 30570906.

Lifang $\mathrm{Jin}^{1,2,3,4, *}$, Shaohui $\mathrm{Ji}^{1,2,3,{ }^{*}}$, Xianghui Tang ${ }^{1,3}$,
Xiangyu Guo ${ }^{1,2,3}$, Yongqing $\mathrm{Lu}^{1,2,3}$, Hongwei Chen ${ }^{1,2,3}$, Hongkui Deng, ${ }^{5}$ Qi Zhou ${ }^{6}$, Weizhi Ji ${ }^{1,3,7}$

${ }^{I}$ Kunming Primate Research Center, and Kunming Institute of Zoology, Chinese Academy of Sciences, Kunming, Yunnan, China; ${ }^{2}$ Graduate School, Chinese Academy of Sciences, Beijing, China; ${ }^{3} Y u n n a n$ Key Laboratory of Animal Reproductive Biology, Kunming, Yunnan, China: ${ }^{4}$ College of Life Sciences, Shaoxing University, Zhejiang, China, ${ }^{5}$ Key Laboratory of Cell Proliferation and Differentiation of the Ministry of Education, College of Life Sciences, Peking University, Beijing, China; ${ }^{6}$ Institute of Zoology, Chinese Academy of Sciences, Beijing, China, ${ }^{7}$ Kunming Biomed International

*These two authors contributed equally to this work.

\section{References}

1 Theise ND. Liver stem cells: prospects for treatment of inherited and acquired liver diseases. Expert Opin Biol Ther 2003; 3:403-408.

2 Laurson J, Selden C, Hodgson HJ. Hepatocyte progenitors in man and in rodents--multiple pathways, multiple candidates. Int J Exp Pathol 2005; 86:1-18.

3 Shafritz DA, Oertel M, Menthena A, Nierhoff D, Dabeva MD. Liver stem cells and prospects for liver reconstitution by transplanted cells. Hepatology 2006; 43:S89-S98.

4 Azuma H, Hirose T, Fujii H, et al. Enrichment of hepatic progenitor cells from adult mouse liver. Hepatology 2003; 37:1385-1394.

5 Schmelzer E, Zhang L, Bruce A, et al. Human hepatic stem cells from fetal and postnatal donors. J Exp Med 2007; 204:1973-1987.

6 Tsuchiya A, Heike T, Fujino H, et al. Long-term extensive expansion of mouse hepatic stem/progenitor cells in a novel serum-free culture system. Gastroenterology 2005; 128:20892104.

7 Nierhoff D, Ogawa A, Oertel M, Chen YQ, Shafritz DA. Purification and characterization of mouse fetal liver epithelial cells with high in vivo repopulation capacity. Hepatology 2005; 42:130-139.

8 Nitou M, Sugiyama Y, Ishikawa K, Shiojiri N. Purification of fetal mouse hepatoblasts by magnetic beads coated with monoclonal anti-e-cadherin antibodies and their in vitro culture. Exp Cell Res 2002; 279:330-343.

9 Strick-Marchand H, Weiss MC. Inducible differentiation and morphogenesis of bipotential liver cell lines from wild-type mouse embryos. Hepatology 2002; 36:794-804.

10 Dan YY, Riehle KJ, Lazaro C, et al. Isolation of multipotent progenitor cells from human fetal liver capable of differentiating into liver and mesenchymal lineages. Proc Natl Acad Sci USA 2006; 103:9912-9917.

(Supplementary Information is linked to the online version of the paper on the Cell Research website.) 